\title{
Indocyanine green guided surgery for pancreatic surgery
}

\author{
Nicolae BACALBASA ${ }^{1,2,3}$, Irina BALESCU ${ }^{4}$, Camelia C. DIACONU ${ }^{1,5}$, Laura ILIESCU ${ }^{1,6}$, \\ Mihaela VILCU ${ }^{1,7}$, Lucian POP ${ }^{8}$, Mihai DIMITRIU ${ }^{1,9}$, Cristian BALALAU ${ }^{1,10}$, \\ Alexandru FILIPESCU ${ }^{1,11}$, Carmen SAVU ${ }^{12}$, Cornel SAVU ${ }^{1,13}$, Emil BELU ${ }^{14}$, Iulian BREZEAN ${ }^{1,7}$ \\ 1"Carol Davila" University of Medicine and Pharmacy, Bucharest, Romania \\ 2Department of Obstetrics and Gynecology, "I. Cantacuzino" Clinical Hospital, Bucharest, Romania \\ ${ }^{3}$ Department of Visceral Surgery, Center of Excellence in Translational Medicine \\ Fundeni Clinical Institute, Bucharest, Romania \\ ${ }^{4}$ Department of Surgery, Ponderas Academic Hospital, Bucharest, Romania \\ ${ }^{5}$ Department of Internal Medicine, University Emergency Hospital Bucharest, Bucharest, Romania \\ ${ }^{6}$ Department of Internal Medicine, Fundeni Clinical Institute, Bucharest, Romania \\ 7Department of Surgery, "I. Cantacuzino" Clinical Hospital, Bucharest, Romania \\ 8"Alessandrescu-Rusescu" National Institute of Mother and Child Health, \\ Fetal Medicine Excellence Research Center, Bucharest, Romania \\ ${ }^{9}$ Department of Obstetrics and Gynecology, "Sf. Pantelimon" Emergency Clinical Hospital, \\ Bucharest, Romania \\ ${ }^{10}$ Department of Surgery, Pantelimon Clinical Hospital, Bucharest, Romania \\ ${ }^{11}$ Department of Obstetrics and Gynecology, Elias Emergency Hospital, Bucharest, Romania \\ ${ }^{12}$ Department of Anesthesiology, Fundeni Clinical Institute, Bucharest, Romania \\ ${ }^{13}$ Department of Thoracic Surgery, "Marius Nasta" Institute of Pneumonology, Bucharest, Romania \\ ${ }^{14}$ Department of Oncology, "Prof. Dr. Al. Trestioreanu" Institute of Oncology Bucharest Romania
}

\begin{abstract}
Indocyanine green guided surgery has gained significant popularity in the last decade in different areas including colorectal, gynecological or even hepatic surgery. Moreover, recently published papers came to demonstrate the utility of the method in pancreatic surgery, providing a better identification of pancreatic lesions and bringing significant information regarding the local anatomy. The current paper represents a literature review of the most relevant studies published so far on this issue.
\end{abstract}

Keywords: pancreatic tumors, indocyanine green, infrared guided surgery

\section{Abbreviation}

ICG = Indocyanine green

\section{INTRODUCTION}

Indocyanine green (ICG) and infrared guided surgery gained significant popularity in the last decades, multiple benefits being described so far. Therefore, ICG administration followed by infrared imaging can be safely used in order to obtain significant information regarding anatomical details such as vasculariza- 
tion details during resection and anastomosis, sentinel lymph node identification in early stage cancers or even information regarding the intrahepatic blood flow distribution $(1,2)$. All these information seem to significantly improve the outcomes of surgically treated patients.

\section{THE PRINCIPLE OF THE METHOD}

Approved by the Food and Drug Administration for clinical and research purposes in humans since 1956, ICG is in fact a fluorescent dye which, when injected intravenously it binds to plasma proteins, is excited between 750 and $800 \mathrm{~nm}$ and is detected at a maximum peak of $832 \mathrm{~nm}$ (3-5). In this respect, ICG guided surgery has been widely used so far in plastic surgery, oncogynecologic surgery and surgical oncology with multiple purposes $(1,2)$.

\section{ICG UTILITY IN PANCREATIC SURGERY}

Initially, ICG guided surgery in hepato-bilio-pancreatic surgery has been proposed in order to diminish the risk of intraoperative incidents during difficult laparoscopic cholecystectomy. Later on, the method proved to be efficient in order to evaluate the liver function, to guide anatomical liver resection or to identify liver metastases with different origins (6).

Utility of ICG in pancreatic surgery is mainly based on the rich vascularization of this organ, a blood flow ranging between $38.4 \mathrm{ml} / \mathrm{min} / 100 \mathrm{ml}$ to up to $356 \mathrm{ml} /$ $\mathrm{min} / 100 \mathrm{ml}$ being reported so far; meanwhile, pancreatic tumors present a poorer vascularization and therefore, intravenous administration of ICG followed by infrared inspection will offer strong contrast between the healthy pancreatic tissue and the poor perfused tumoral pancreatic tissue (7). Moreover, ICG administration can offer real time information regarding the vascularization of the pancreatic stump and might therefore decrease the risk of anastomotic leakage (8). The first case report which presented the utility of ICG guided pancreatic resection comes from the Korean team conducted by Sung Hyun Kim et al. and was published in 2017; the paper reported the case of a 28-year-old patient in whom a $4.5 \mathrm{~cm}$ solid pseudopapillary neoplasm was discovered at the level of the pancreatic tail. The patient was submitted to ICG guided laparoscopic surgery which proved to be efficient in identifying the tumor and providing complete resection (7). The largest study which came to demonstrate the utility of intravenous administration for achieving negative resection margins in pancreatic head adenocarcinoma was conducted by Rho et al. and published in 2018. The study included 37 patients submitted to pancreaticoduodenectomy for pancreatic head can- cer; in 10 cases ICG guided surgery was used. The authors demonstrated the superiority of ICG guided surgery in order to achieve negative retroperitoneal resection margins; meanwhile no significant differences were observed in regard to the mean operative time, mean blood loss and postoperative pancreatic related complications (9).

Meanwhile, other recent studies came to demonstrate the effectiveness and utility of ICG intravenous administration in pancreatic neuroendocrine tumors, based on the fact that a higher affinity of neuroendocrine tumoral cells for ICG exists (when compared to pancreatic cells). The largest study which came to demonstrate utility of ICG in neuroendocrine pancreatic tumors detection has been published in 2017 by Paiella and included 10 patients submitted to surgery between March 2016 and October 2016 (10). In all cases, 5 boli of 5 mg each of ICG were injected at every 60 seconds, most tumors being identified after the second ICG bolus, with a mean latency time of 80 seconds and a mean visibility time of 220 seconds. Moreover, the peak of visualization of the tumoral mass was reached at 20 minutes after the administration of the last ICG bolus. The first intravenous ICG bolus was administrated after sectioning the gastrocolic ligament and exposing the anterior surface of the gland. In order not to diminish the ICG inflow at the level of the pancreatic gland, the authors decided not to ligate the splenic vessels until the moment of ICG administration even if a splenopancreatectomy was planned. Meanwhile, the completeness of resection was also verified by intraoperative ultrasound. After ICG administration in two out of the 10 cases additional lesions were found and were separately resected; however in both cases the histopathological studies demonstrated that these additional lesions were in fact lymph nodes. As for the other lesions, the presence of neuroendocrine tumors was confirmed in all cases by the histopathological studies (10). These data came to demonstrate the correctitude of the authors' supposition that, due to the fact that ICG is bounded by the plasmatic proteins, it will reach a higher concentration in blood and, consecutively, a higher amount of ICG will be seen in intensively vascularized tissues such as neuroendocrine tumoral tissues (10).

Another utility of ICG administration in pancreatic surgery is related to the lymphatic drainage mapping in order to establish the extent of the lymph node dissection. Therefore, in a study conducted by Hirono et al., the authors injected ICG at the level of the anterior pancreatic surface in 10 cases and respectively in the posterior surface in other 10 cases and observed that the main lymphatic patterns of spread were to the para-aortic region in 17 cases and respectively to the superior mesenteric artery in 4 cases; interestingly, the 
meantime for reaching the superior mesenteric artery region was higher when compared to the time for reaching the para-aortic region; these data enabled the authors to consider that during pancreatoduodenectomy lymph node dissection at the level of the superior mesenteric artery might have similar outcomes when compared to para-aortic lymph node dissection (11).

Similarly to colorectal cancer, intravenous administration of ICG during pancreatic surgery also seems to provide the advantage of identification of small liver metastases which would be otherwise undetectable; therefore the intraoperative intravenous administration of ICG seems to increase the rate of detection of liver metastases and to avoid in this way performing major surgical procedures in cases who already present systemic disease (12).

Another interesting utility of ICG in pancreatic resections is represented by the possibility of real time visualization of the duodenal arterial supply as well as of the biliary tract; based on these properties, intravenous ICG administration proved to be an effective method in order to decrease the intraoperative and postoperative complications which might develop during duodenum preserving pancreatic head resection (also known as Beger procedure). Therefore, intravenous administration of ICG provides a better visualiza- tion of the gastroduodenal and anterosuperior pancreatic duodenal artery as well as of the common bile duct, decreasing in this way the risk of iatrogenic injury of these structures (13).

\section{CONCLUSIONS}

Although ICG administration in pancreatic surgery has been only recently investigated, the method seems to be extremely efficient in order to provide the identification of different types of pancreatic lesions, to exclude the presence of distant metastases such as the hepatic ones, to provide a complete tumoral resection, to achieve a more specific lymph node dissection and to preserve certain vascular and biliary structures. Preliminary results published so far seem to be extremely encouraging; however, larger studies are still needed in order to explore the benefits of intravenous or intrapancreatic injection of ICG and infrared guided surgery.

\section{Acknowledgements}

This work was supported by the project entitled „Multidisciplinary Consortium for Supporting the Research Skills in Diagnosing, Treating and Identifying Predictive Factors of Malignant Gynecologic Disorders", project number PN-III-P1-1.2-PCCDI2017-0833.

Conflict of interest: none declared

\section{REFERENCES}

1. Diana M. Enabling precision digestive surgery with fluorescence imaging. Transl Gastroenterol Hepatol. 2017;2:97.

2. Gioux S, Choi HS, Frangioni JV. Imageguided surgery using invisible near-infrared light: fundamentals of clinical translation. Mol Imaging. 2010;9:237-255.

3. Alander JT, Kaartinen I, Laakso A et al. A review of indocyanine green fluorescent imaging in surgery. Int J Biomed Imaging. 2012;2012:940585.

4. Yoneya S, Saito T, Komatsu Y, Koyama I, Takahashi K, Duvoll-Young J. Binding properties of indocyanine green in human blood. Invest Ophthalmol Vis Sci. 1998;39:1286-90.

5. Mordon S, Devoisselle JM, Soulie-Begu S, Desmettre T. Indocyanine green: Physicochemical factors affecting its fluorescence in vivo. Microvasc Res. 1998;55:146-152.

6. Baiocchi GL, Diana M, Boni L. Indocyanine green-based fluorescence imaging in visceral and hepatobiliary and pancreatic surgery: State of the art and future directions. World J Gastroenterol. 2018;24:2921-30.

7. Kim SH, Rho SY, Kang CM. Indocyanine Green-Fluorescent Pancreatic PerfusionGuided Resection of Distal Pancreas in Solid Pseudopapillary Neoplasm: Usefulness and Feasibility During Pancreaticobiliary Surgery. J Minim Invasive Surg. 2018;21:43-45.

8. Strasberg SM, McNevin MS. Results of a technique of pancreaticojejunostomy that optimizes blood supply to the pancreas. J Am Coll Surg. 1998;187:591-596.

9. Rho SY, Kim JS, Chong JU et al. Indocyanine Green Perfusion ImagingGuided Laparoscopic Pancreaticoduodenectomy: Potential Application in Retroperitoneal Margin Dissection. J Gastrointest Surg. 2018;22:1470-1474.

10. Paiella S, De Pastena M, Landoni L et al. Is there a role for near-infrared technology in laparoscopic resection of pancreatic neuroendocrine tumors? Results of the COLPAN "colour-and-resect the pancreas" study. Surg Endosc. 2017;31:4478-84.

11. Hirono S, Tani M, Kawai M et al. Identification of the lymphatic drainage pathways from the pancreatic head guided by indocyanine green fluorescence imaging during pancreaticoduodenectomy. Dig Surg. 2012;29:132-139.

12. Shirakawa S, Toyama H, Kido M, Fukumoto T. A prospective single-center protocol for using near-infrared fluorescence imaging with indocyanine green during staging laparoscopy to detect small metastasis from pancreatic cancer. BMC Surg. 2019;19:165.

13. Chen $\mathrm{S}$, Gao P, Cai $\mathrm{H}$ et al. Indocyanine Green-Enhanced Fluorescence in Laparoscopic Duodenum-Preserving Pancreatic Head Resection: Technique with Video. Ann Surg Oncol (2020). 